\title{
Etica, trascendenza e sostenibilità ${ }^{1}$
}

\author{
Ethics, transcendence and sustainability
}

Cardinal Gianfranco Ravasi

\section{Resumo}

Tendo presente a realidade hodierna e, sobretudo, o desafio dominante aos nossos dias, de salvar o planeta, a nossa Casa Comum, muitos passos devem ser ainda percorridos, em consonância com a história já realizada até agora. A colaboração entre crentes e não crentes se torna cada dia mais necessária e urgente. Por isso, deve-se refletir sobre alguns temas fundamentais que nos ajudam a caminhar juntos, como: ética, verdade, transcendência, imanência, desenvolvimento, sustentabilidade, pluralismo cultural, natureza humana, laicidade, reciprocidade, doação etc. O presente ensaio quer oferecer uma pequena contribuição neste Ano da Misericórdia à proposta que nos é apresentada pelo Papa Francisco para que se possa dialogar com o mundo, sobretudo a partir das várias experiências dos "Pátios dos Gentios" que muitas dioceses da Igreja católica e instituições leigas estão realizando no mundo, buscando sempre o diálogo com a sociedade em geral, seja com os crentes seja com os não crentes. A finalidade é aquela de refletir juntos para encontrar caminhos possíveis para salvar o nosso planeta, a partir de uma ética que tenha presente a sustentabilidade e também respeite o transcendente. Seguramente no futuro a humanidade conhecerá um desenvolvimento maior que aquele hodierno, mas o grande desafio é aquele de efetuá-lo sempre em maneira responsável e sustentável, em vista do bem comum e não de poucos

\footnotetext{
${ }^{1}$ Questo saggio contiene due conferenze pronunciate dal Cardinale Gianfranco Ravasi nella Città di Rio de Janeiro, Brasile, durante il "Cortile dei Gentili", ivi chiamato "Patio dos Encontros": la prima il 7 aprile 2016, presso il Teatro Municipale, nel "Pátio dos Encontros", in un dialogo tra credenti e non credenti; la seconda 1'8 aprile 2016, presso il Salão da Pastoral della PUC-Rio, nella Lectio Magistralis (Aula Magna) in apertura dell'Anno Accademico 2016, per la Comunità Accademica della stessa Università. I due testi sono stati messi qui in sequenza, rispettandone i titoli e le divisioni interne. Proprio per questo il testo non contiene un'introduzione né una conclusione generale, se non quelle di ciascuna delle due parti del testo.
} 
privilegiados. Então, é indispensável que crentes e não crentes podem e devem trabalhar juntos. O horizonte será sempre aquele de um profícuo diálogo e também de um respeito em relação ao mistério do Transcendente presente na Criação para o bem de todos os homens.

Palavras-chave: Ética. Transcendência. Sustentabilidade. Ecologia. Desenvolvimento.

\section{Sommario}

Avendo presente la realtà attuale e soprattutto la sfida, dominante ai nostri giorni, di salvare il pianeta, la nostra Casa Comune, molti passi devono essere ancora percorsi, in consonanza con la storia già finora realizzata. In questo ambito, la collaborazione tra credenti e non credenti diventa ogni giorno più necessaria e urgente. Per questo si deve riflettere su alcuni temi fondamentali che ci aiutano a camminare insieme, come: etica, verità, trascendenza, immanenza, sviluppo, sostenibilità, pluralismo culturale, natura umana, laicità, reciprocità e donazione etc. Il presente saggio vuol offrire un piccolo contributo in questo Anno della Misericordia alla proposta avanzata da Papa Francesco perché si possa dialogare con il mondo, soprattutto a partire delle varie esperienze del "Cortile dei Gentili" che molte diocesi della Chiesa cattolica e istituzioni laiche stanno realizzando nel mondo, cercando sempre il dialogo con la società in generale, sia con i credenti sia con i non credenti. La finalità è quella di riflettere insieme per trovare vie possibili per salvare il nostro pianeta, a partire da un'etica che abbia presente la sostenibilità e rispetti anche il trascendente. Sicuramente in futuro l'umanità conoscerà uno sviluppo maggiore di quello odierno, ma la grande sfida è quella di attuarlo sempre in maniera responsabile e sostenibile, in vista del bene comune e non di pochi privilegiati. È, quindi, indispensabile che credenti e non credenti possano e debbano lavorare insieme. L'orizzonte sarà sempre quello di un proficuo dialogo e anche di un rispetto verso il mistero del trascendente presente nel creato per il bene di tutti gli uomini.

Parole-chiave: Etica. Trascendenza. Sostenibilità. Ecologia. Sviluppo. 


\begin{abstract}
In view of the present reality and, above all, the main challenge nowadays that is to save the Planet, our Common Home, many steps must yet be taken, but in accordance with the history already made so far. The collaboration between believer and non-believers becomes ever more necessary and urgent with each day. Therefore, it is also necessary to reflect upon certain fundamental topics that help us walk together, such as: ethics, truth, transcendence, immanence, development, sustainability, cultural pluralism, human nature, common platform, laicism, reciprocity, donation etc. This essay offers a small contribution on this Year of Mercy to the request presented to us by Pope Francis to dialogue with the world, especially from the various experiences of the "Courtyards of the Gentiles" that many dioceses of the catholic Church and lay institutions are performing in the world, always seeking dialogue with the society in general, with believers or with non-believers. The purpose is to reflect together to find possible ways to save our planet, from an ethical starting point mindful of sustainability and that also respects the Transcendent. Surely in the future mankind will know more development than in the present-day, but the greatest challenge is that of achieving it always in a responsible and sustainable manner in view of the common good and not of the privileged few. Therefore, it is imperative that believers and non-believers can and must work together. The horizon will always be that of a fruitful dialogue and also that of respect in relation to the mystery of the Transcendent present in the work of Creation for the good of all men.
\end{abstract}

Keywords: Ethics. Transcendence. Sustainability. Ecology. Development.

\title{
1. Etica e trascendenza
}

Nella prefazione al suo Tractatus logico-philosophicus (1921) il filosofo viennese Ludwig Wittgenstein ${ }^{2}$, illustrando lo scopo della sua ricerca, affermava che era sua intenzione investigare i contorni di un'isola, ossia l'uomo circoscritto e limitato. Ma ciò che aveva alla fine scoperto erano le frontiere dell'oceano. La metafora è chiara: se si percorre il litorale di un'isola, guardando solo al suo tracciato terrestre, si riesce a computarla, definirla,

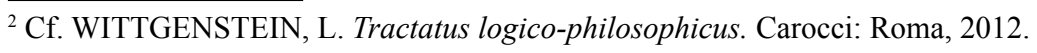


identificarla. Ma se lo sguardo si volge verso l'altro versante della costa, si intuisce il distendersi del mare infinito. In sostanza nell'essere umano si ha un intreccio tra la finitudine e l'infinito, tra un contingente sperimentale e un oltre altrettanto significativo ma più imponderabile.

\subsection{Tra immanenza e trascendenza}

Nella storia del pensiero si sono, così, confrontati due modelli estremi. C'è chi ha optato solo per l'isola, scegliendo le varie forme di immanentismo, coi loro corollari gnoseologici, etici, esistenziali, persino sociali. Essi potevano anche esasperarsi, come nel razionalismo, nel materialismo, nel fenomenismo, nel relativismo, nel soggettivismo, nel secolarismo, nello stesso postumanesimo e in certi approcci tecnologici radicali. L'antropologia risulterebbe, così, amputata da ogni dimensione trascendente, fissandosi solo su un orizzonte privo di verticalità. C'è, però, anche l'estremo opposto del trascendentalismo, che si protende soltanto verso l'oceano, il mistero, l'infinito e l'eterno, talora decollando dalla realtà verso il cielo purissimo ma astratto del dogmatismo, del fondamentalismo, dell'ideologismo e persino dell'assolutismo sacrale.

Bisogna, però, ricordare che un'ampia porzione della ricerca filosofica e soprattutto teologica si è invece sforzata di tenere insieme "simbolicamente" le due sponde, la terrena e l'infinita, combattendo ogni radicalismo esclusivista. Certo, l'equilibrio è delicato perché deve tenere intrecciate tra loro dimensioni dotate di una loro autonomia come la fisica e la metafisica, la prassi e l'etica, la storia e l'eterno. Già la cultura classica, soprattutto greca, è stata veramente esemplare nel compiere questa operazione "sim-bolica". Proponiamo solo qualche esempio in modo molto semplificato.

Platone $^{3}$ svela nel suo Iperuranio la presenza dei tre grandi trascendentali del Vero, del Bene e del Bello: essi si irradiano e vengono partecipati divenendo il fondamento di ogni ente, di ogni razionalità, di ogni etica. Aristotele ${ }^{4}-$ seguito poi da s. Tommaso d'Aquino ${ }^{5}$ - punterà, invece, a un vertice unico

${ }^{3}$ Cf. REALE, G. (Curatore). Platone, Opere Complete, Tutti gli scritti. Milano: Bompiani, 2000.

${ }^{4}$ Cf. MAZZARELLI, C. (Curatore). Aristotele, Etica Nicomachea. Milano: Bompiani, 2000; REALE, G. (Curatore). Aristotele, Metafisica. Milano: Bompiani, 2000.

${ }^{5}$ Cf. FIORENTINO, F. (Curatore). Tommaso d'Aquino, Sulla Verità. Milano: Bompiani, 2005; CENTI, T. S. (Curatore). Tommaso d'Aquino (san), Somma contro i gentili. 3 volumni. Bologna: Edizione Studio Domenicano, 2001; BARZAGHI, G. (Curatore). Tommaso d'Aquino 
supremo, l'Essere, primo motore perfetto e immobile, principio però dell'uno, del vero, del bene e "pensiero del pensiero" di ogni essere umano. Plotino ${ }^{6}$, col neoplatonismo e con la successiva riflessione agostiniana ${ }^{7}$, porrà all'apice il Nous trascendente, una Mente che è Essere e Bene divino dalla quale procede la totalità degli esseri, in una sequenza decrescente di perfezione fino al livello estremo ove l'essere si dissolve nel nulla.

Tutte queste concezioni, pur nelle loro diversità e variazioni, cercano di comporre un nesso stretto tra il trascendente e l'immanente. Se vogliamo stare al livello morale, bisogna riconoscere che, quanto più ci si distacca dal bene trascendente - attraverso le scelte negative della libertà umana - , tanto più cresce e imperversa l'immoralità, cioè l'empietà, la falsità, l'odio, la bruttura etica e la bruttezza estetica. Questa prospettiva ha una sua rappresentazione molto suggestiva nell'antropologia della Bibbia che è, pur sempre, il nostro "grande codice culturale". Bisogna innanzitutto ricordare che, soprattutto per il cristianesimo (ma i prodromi sono già nella "rivelazione storica" dell'Antico Testamento), fondamentale è il legame tra trascendenza e immanenza.

È ciò che esprime la dottrina cristiana dell'Incarnazione, luminosamente definita nel celebre prologo del Vangelo di Giovanni. Da un lato, c'è l'affermazione del Lógos che è «in principio, presso Dio ed è Dio... Tutto è stato fatto per mezzo di lui e senza di lui nulla è stato fatto di ciò che esiste» (Giovanni 1,1-3). D'altro lato, c'è la convinzione che «il Lógos divenne sarx» (Giovanni 1,14), cioè carnalità, fragilità, caducità, immanenza finita e mortale. Jorge Luis Borges riprendeva e rielaborava liberamente ma incisivamente il passo evangelico nella sua poesia Giovanni 1,14: «Io so che sono l'È, il Fu e il Sarà / accondiscendo al linguaggio / che è tempo successivo e simbolo [...]. / Vissi stregato, prigioniero di un corpo / e di un'umile anima [...]. / Fui amato, compreso, esaltato e appeso a una croce» ${ }^{8}$.

Ora, per tornare al tema della trascendenza etica, a livello biblico è emblematica la scena di esordio della stessa Bibbia: l'uomo e la donna sono

(san), Somma Teologica. Edizione Integrale: Opera completa in 4 volumi. Bologna: Edizione Studio Domenicano, 2014.

${ }^{6}$ Cf. BAILONE, G. Plotino. Pistoia: Editrice Petite Plaisance, 2010.

${ }^{7}$ Cf. BIEIRWALTES, W. Agostino e il Neoplatonismo Cristiano. Milano: Vita e Pensiero, 1991; RIST, J. M. Agostino, Il Battesimo del Pensiero Antico. Milano: Vita e Pensiero, 1997.

${ }^{8}$ Questa poesia si trova nel Commento di Jorge Luis Borges al passo del Vangelo di Giovanni 1,14, in occasione della Pasqua del 2011, a Buenos Aires, Argentina. Il testo completo in italiano si trova anche in internet. 
collocati, nei cc. 2-3 della Genesi, all'ombra «dell'albero della conoscenza del bene e del male», un albero intoccabile, cioè trascendente e preesistente, fisso nella sua entità che precede ed eccede la pur reale libertà umana. Quell'albero diventa, quindi, il simbolo della morale. Certo, la scelta libera della persona può accogliere quella determinazione trascendente del bene e del male, oppure, strappandone il frutto, decidere in proprio ciò che è bene e male, relativizzando così l'assolutezza dei valori morali. La stessa rappresentazione è presente nel Decalogo che è proposto dall'alto della vetta del Sinai, dalla voce divina, simbolo della trascendenza dell'etica che è in sé codificata. Ma anche in questo caso, decisiva è la libertà umana che può accogliere, custodire e osservare la legge morale, oppure ricomporla a suo piacimento, come accade nell'episodio altrettanto simbolico del vitello d'oro (Esodo 32).

\subsection{Il concetto di verità}

Come è evidente, in questa concezione etica è fondamentale il nesso e l'interazione tra oggettivo e soggettivo, tra precetto e opzione, tra assoluto e libertà, tra trascendenza e immanenza. Un legame molto delicato e complesso che è, comunque, l'anima stessa della morale classica e giudeo-cristiana e che ha un valore parallelo in un altro ambito affine a cui vorremmo ora accennare perché altrettanto significativo. Intendiamo riferirci alla categoria verità. Se noi seguiamo il percorso culturale di questi ultimi secoli, infatti, possiamo dire che il concetto di verità è diventato sempre più immanente $\mathrm{e}$ soggettivo fino ad arrivare al "situazionismo" del secolo scorso. Si pensi, ad esempio, alla frase significativa e spesso citata, attinta al Leviathan del filosofo inglese secentesco Hobbes ${ }^{9}$ : Auctoritas, non veritas facit legem. In ultima analisi è, questo, il principio del contrattualismo, secondo il quale l'autorità, sia civile sia religiosa, può decidere la norma e, quindi, indirettamente la verità, in base alle convenienze della società e ai vantaggi del potere secondo le circostanze contingenti.

Tale concezione fluida della verità è ormai abbastanza acquisita nella cultura contemporanea. Basti pensare all'antropologia culturale. Infatti, il filosofo francese Michel Foucault ${ }^{10}$, studiando le diverse culture e le loro variabili comportamentali, invitava caldamente ad accentuare questa

\footnotetext{
${ }^{9}$ Cf. SANTI, R. (Curatore). Tommas Hobbes, Leviatano. Milano: Bompiani, 2001.

${ }^{10}$ CF. FOUCAULT, M. Il coraggio della verità. Roma: Feltrinelli, 2011.
} 
dimensione soggettiva e mutevole della verità, simile a una medusa cangiante, che cambia aspetto continuamente a seconda dei contesti e delle circostanze. Questo soggettivismo è sostanzialmente ciò che Benedetto XVI ha chiamato "relativismo" "11, ed è curioso notare come la pensatrice americana, Sandra Harding ${ }^{12}$, facendo il verso a una celebre frase del Vangelo di Giovanni $(8,32$ : «La verità vi farà liberi»), affermava al contrario in un suo saggio che «la verità non vi farà liberi». Essa, infatti, viene concepita come una cappa di piombo oppressiva, come una pre-comprensione, come una sterilizzazione della dinamicità e dell'incandescenza del pensiero umano.

Tutte le religioni, e in particolare il cristianesimo, hanno invece una concezione trascendente della verità: la verità ci precede e ci supera; essa ha un primato di illuminazione, non di dominio. Anche se Theodor Adorno l'aveva applicata soprattutto alla felicità, è suggestiva una sua espressione tratta dai Minima moralia ${ }^{13}$. Il filosofo tedesco, parlando della verità e comparandola appunto alla felicità, dichiara: «La verità non la si ha, ma vi si è», cioè si è immersi in essa. Robert Musil, nel suo famoso romanzo L'uomo senza qualita ${ }^{14}$, al protagonista fa dire una frase interessante: «La verità non è come una pietra preziosa che si può mettere in tasca, bensì è come un mare nel quale ci si immerge».

Si tratta, fondamentalmente, della classica concezione platonica espressa nel Fedro ${ }^{15}$ mediante l'immagine della "pianura della verità": la biga dell'anima corre su questa pianura preesistente ed esterna per conoscerla e conquistarla. Proprio per questo, nella Apologia di Socrate ${ }^{16}$, lo stesso filosofo affermava: «Una vita senza ricerca non merita di essere vissuta». Ė questo l'itinerario da compiere nell'orizzonte "dato" e, quindi, trascendente della verità. Da tale punto di vista le religioni sono nette: la verità ha un primato che ci supera, la verità è appunto trascendente, e compito dell'uomo è essere pellegrino all'interno dell'assoluto della verità. Per questo in esse si considera divina la verità: non per nulla il cristianesimo applica a Cristo l'identificazione con la verità per eccellenza (Giovanni 14,6: «Io sono la Via, la Verità, la Vita»).

\footnotetext{
${ }^{11}$ Cf. BENEDETTO XVI. Lettera Enciclica Caritas in Veritate. Roma: Editrice Vaticana, 2009.

${ }^{12}$ HARDING, S. Science and social inequality, Feminism and Postcolonial Issues. Chiacago: University Ilinois Press, 2006.

${ }^{13}$ Cf. ADORNO, T. W. Minima Moralia. Meditazioni della vita offesa. Torino: Einaudi, 2005.

${ }^{14}$ Cf. MUSIL, R. L'uomo senza qualità. Torino: Einaudi, 2005.

${ }^{15}$ Cf. MATI, S. (Curatore). Platone, Fedro. Roma: Feltrinelli, 2008.

${ }^{16}$ Cf. REALE, G. (Curatore). Platone, Apologia di Socrate. Roma: Feltrinelli, 2000.
} 


\subsection{Il concetto di "natura umana"}

Ma ritorniamo alla specificità del nostro tema con un'ultima riflessione su un'altra categoria capitale per quanto concerne il rapporto tra etica e trascendenza. Accade spesso che nelle esperienze del cosiddetto "Cortile dei Gentili" - cioè del dialogo tra credenti e non credenti da svolgere nello spazio aperto e libero della discussione fuori del Tempio e del Palazzo - ci si interroghi sulla possibilità o meno di avere una piattaforma comune di incontro. Il discorso punta, allora, sul concetto di natura umana: essa può essere concepita in senso metafisico e quindi trascendente, oppure deve ridursi a una mera proceduralità sociale, priva di indicazioni morali "oggettive"?

Ebbene, in questi ultimi tempi attorno a una tale categoria antropologica basilare si è abbattuta una bufera che ne ha scosso le fondamenta: basti solo pensare al "politeismo dei valori" registrato da Weber ${ }^{17}$ o anche al puro e semplice pluralismo culturale. La domanda, allora, è questa: è possibile recuperare un concetto condiviso di "natura" antropologica che superi la mera fenomenologia delle possibili opzioni, che abbia quindi un fondamento che trascenda la mutabilità dei costumi e che quindi impedisca di scivolare nelle sabbie mobili del già evocato relativismo, cioè di una molteplicità sfaldata $\mathrm{e}$ babelica?

Nella storia del pensiero occidentale attorno a questa categoria possiamo individuare come due grandi fiumi interpretativi, dotati di tante anse, affluenti e ramificazioni ma ben identificabili nel loro percorso. Il primo ha la sua sorgente ideale nella filosofia aristotelica che - come già si diceva - per formulare il concetto di natura umana ha attinto alla matrice metafisica dell'essere. La base è, perciò, oggettiva e trascendente, iscritta nella realtà stessa della persona, e funge da stella polare necessaria per l'etica. Questa concezione, dominante per secoli nella filosofia e nella teologia, è icasticamente incisa nel motto della filosofia scolastica medievale Agere sequitur esse, il dover essere nasce dall'essere, l'ontologia precede la deontologia.

Questa impostazione piuttosto granitica e fondata su un basamento solido ha subito in epoca moderna una serie di picconate, soprattutto quando a partire da Cartesio ${ }^{18}$ e dal riconoscimento dell'importanza della soggettività (cogito, ergo sum) - si è posta al centro la libertà personale. Si è diramato,

\footnotetext{
${ }^{17}$ Cf. GHIA, F. Max Weber, Il Politeismo dei valori. Brescia: Morcelliana, 2010.

${ }^{18}$ Cf. CAMPI, R. (Curatore). René Descartes, Discorso del Metodo. Milano: Rizzolli, 2010.
} 
così, un altro fiume che ha come sorgente il pensiero kantiano: la matrice ora è la ragione pratica del soggetto col suo imperativo categorico, il "tu devi". Al monito della "ragione", della legge morale incisa nella coscienza, si unisce la "pratica", cioè la determinazione concreta dei contenuti etici, guidata da alcune norme generali, come la "regola d'oro" ebraica e cristiana («non fare all'altro ciò che non vuoi sia fatto a te»e " "fa' all'altro ciò che vuoi ti si faccia») o come il principio "laico" del non trattare mai ogni persona come mezzo bensì come fine.

La metamorfosi è significativa: al trascendentale ontologico aristotelicotomistico si sostituisceil trascendentale gnoseologico, la cosiddetta "conoscenza a priori" o ragione universale. La trascendenza è, dunque, affermata ma è assegnata alla legge interna dello spirito umano. Essa regola l'esperienza e le varie conoscenze e scelte etiche parziali personali. Famosa è la finale della Critica della ragion pratica $^{19}$ (1788), ove si afferma una duplice trascendenza, quella fisico-cosmica e quella morale umana: «Due cose riempiono l'animo di ammirazione e di riverenza sempre nuove e crescenti quanto più spesso e a lungo il pensiero vi si sofferma: il cielo stellato sopra di me e la legge morale in me (der bestirnte Himmel über mir und das moralische Gesetz in mir)».

Frantumata da tempo la metafisica aristotelica, si è però assistito nell'epoca contemporanea anche alla dissoluzione della ragione universale kantiana che pure aveva una sua "solidità". Ci si è trovati, così, su un terreno molle, ove ogni fondamento si è sgretolato, ove il "disincanto" ha fatto svanire ogni discorso sui valori, ove la secolarizzazione ha avviato le scelte morali solo sul consenso sociale e sull'utile per sé o per molti, ove il multiculturalismo ha prodotto non solo un politeismo religioso ma anche un pluralismo etico. Al "dover essere" che era stampato nell'essere o nel soggetto si è, così, sostituita solo una normativa procedurale o un'adesione ai mores dominanti, cioè ai modelli comuni esistenziali e comportamentali di loro natura mobili.

È possibile reagire a questa deriva che conduce all'attuale delta ramificato di un'etica variabile così da ricomporre una nuova tipologia di "natura" che conservi un po' delle acque dei due fiumi simbolici sopra evocati senza le rigidità delle loro mappe ideologiche? In parallelo ma anche in autonomia rispetto alla netta concezione della trascendenza teologica ed etica propria della religione, molti ritengono che sia possibile creare un nuovo modello filosofico-morale centrato su un altro assoluto, la dignità della persona,

${ }^{19}$ Cf. GENTILE, G. (Traduttore). Immanuel Kant, Critica della ragion pratica. Testo originale a fronte. Bari: Laterza, 2005. 
còlta nella sua qualità relazionale. Si unirebbero, così, le due componenti dell'oggettività (la dignità) e della soggettività (la persona) legandole tra loro attraverso la relazione all'altro, essendo la natura umana non monadica e chiusa in se stessa ma dialogica, non cellulare ma organica, non solipsistica ma comunionale. È questo il progetto della filosofia personalistica (pensiamo ai contributi di Buber ${ }^{20}$, Lévinas ${ }^{21}$, Mounier ${ }^{22}$, Ricoeur ${ }^{23}$ ).

La natura umana così concepita recupera, allora, una serie di categorie etiche classiche che potrebbero dare sostanza al suo realizzarsi. Proviamo ad elencarne alcune. Innanzitutto la virtù della giustizia che è strutturalmente $a d$ alterum e che il diritto romano aveva codificato nel principio Suum cuique tribuere (o Unicuique suum): a ogni persona dev'essere riconosciuta una dignità che affermi l'unicità ma anche l'universalità per la sua appartenenza all'umanità. Parlavamo sopra di parallelo con la religione: nella stessa linea procede la cultura ebraico-cristiana col Decalogo che evoca i diritti fondamentali della persona alla libertà religiosa, alla vita, all'amore, all'onore, alla libertà, alla proprietà. Nella stessa prospettiva si colloca la citata "regola d'oro".

In sintesi, l'imperativo morale fondamentale si dovrebbe ricostruire partendo da un'ontologia personale relazionale, in pratica dalla figura universale (e cristiana) del "prossimo" e dalla logica dell'amore nella sua reciprocità ma anche nella sua gratuità e donazione. Per spiegarci in termini biblici a tutti noti: «Ama il prossimo tuo come te stesso» (reciprocità), ma anche «non c'è amore più grande di chi dà la vita per la persona che ama» (donazione). Inoltre, in senso più completo, nel dialogo "io-tu" è coinvolto - come suggeriva il filosofo francese Paul Ricoeur ${ }^{24}$ - pure il "terzo", cioè l'umanità intera, anche chi non incontro e non conosco ma che appartiene alla comune realtà umana. Da qui si giustifica, allora, anche la funzione della politica dedicata a costruire strutture giuste per l'intera società. La riflessione attorno a questi temi è naturalmente più ampia e complessa e dovrebbe essere declinata secondo molteplici applicazioni, ma in ultima analisi potrebbe

\footnotetext{
${ }^{20}$ BUBER, M. Il problema dell'uomo. Genova: Marietti, 2013.

${ }^{21}$ Cf. MOSCATO, A. (Curatore). Emmanuel Levinás, L'umanesimo dell'altro uomo. Genova: Il Nuovo Melangolo, 1998.

${ }^{22}$ Cf. CAMPANINI, G. (Curatore). Emmanuel Mounier, Il Personalismo. Roma: AVE, 2004.

${ }^{23}$ Cf. RICOEUR, P. La persona. Brescia: Morcelliana, 1997.

${ }^{24}$ Cf. RICOEUR, P. Sé come un altro. Milano: Jaca Book, 2011; CAPUTO, A. Io e tu. Una dialettica fragile e spezzata. Percorsi con Paul Ricoeur. Bari: Stilo, 2009.
} 
essere fondata su un dato semplice, ossia sulla nostra più radicale, universale e costante identità personale dialogica.

\section{Etica e sostenibilità}

\subsection{Il vocabolo sostenibilità}

È un vocabolo che è divenuto quasi un'insegna dei nostri giorni, fino a trasformarsi purtroppo in uno stereotipo che riempie le bocche ma lascia indifferenti le mani e, quindi, l'impegno. Stiamo parlando della cosiddetta sostenibilità, un termine spesso ripetuto e declinato in varie forme (indice, codice, bilancio di sostenibilità) e che, però, registra all'opposto un dato talora drammatico, quello dello sfruttamento insensato ed egoistico dei beni che Dio ha destinato universalmente all'umanità e che, invece, vengono o accaparrati solo da alcuni o sprecati insensatamente (si pensi solo all'acqua!) o feriti attraverso l'inquinamento e la devastazione ambientale.

\subsection{Il Creato e la sostenibilità}

Proprio in apertura alla Bibbia, nelle pagine dedicate alla creazione, ci sono due asserti fondamentali. Il primo è quello che - a differenza della cultura greca - riconosce il rilievo che la materialità ha anche per la creatura umana: «Il Signore Dio plasmò l'uomo con polvere del suolo e soffiò nelle sue narici un alito di vita e così divenne un essere vivente» (Genesi 2,7). Alla fine della sua esistenza l'uomo «ritorna alla polvere della terra e il soffio vitale ritorna a Dio che lo ha dato» (Qohelet 12,7), «poiché da essa sei stato tratto: polvere tu sei e in polvere tornerai» (Genesi 3,19). Tra la terra e l'umanità c'è, dunque, una radicale sororità, una parentela stretta che, però, spesso dimentichiamo $\mathrm{e}$ violiamo.

Il secondo asserto indica un altro aspetto che ci distingue dalla materialità. Il Creatore, infatti, impone questo impegno all'uomo e alla donna: «Siate fecondi e moltiplicatevi, riempite la terra e soggiogatela, dominate sui pesci del mare, sugli uccelli del cielo e su ogni essere vivente che striscia sulla terra» (Genesi 1,28). La creatura umana riceve da Dio una dignità di sovranità delegata sul creato. In realtà, i due verbi ebraici usati contengono un significato più sfumato e persino suggestivo: kabash - "soggiogare" originariamente rimanda all'insediamento in un territorio che dev'essere 
esplorato e conquistato, mentre radah - "dominare" è il verbo del pastore che guida il suo gregge.

Si tratta, certo, di un primato che purtroppo l'uomo spesso ha esercitato in modo tirannico e non come un compito, che è specificato da un ulteriore dettato del Creatore così formulato: «Il Signore Dio prese l'uomo e lo pose nel giardino di Eden perché lo coltivasse e custodisse» (Genesi 2,15). È interessante notare anche in questo caso che l'attività propria dell'umanità è espressa con due verbi ebraici - 'abad e shamar - che contengono un duplice significato. Il primo è quello esplicito dell'operare, trasformare, investigare e tutelare le potenzialità della natura attraverso l'attività lavorativa e scientifica. Il secondo aspetto è nel fatto che i due verbi indicano anche il "servire" cultuale e 1" "osservare" la legge divina, due componenti fondamentali dell'alleanza tra il Signore e Israele.

C'è, dunque, una sorta di alleanza primaria "naturale" tra il Creatore e l'umanità che si esprime nella tutela e nella trasformazione del creato. Un patto che spesso l'uomo infrange, devastando e occupando brutalmente la terra. È suggestiva una parabola araba che si muove proprio in questa linea. «All' inizio il mondo era un giardino fiorito. Dio, creando l'uomo, gli disse: Ogni volta che compirai un'azione cattiva, io farò cadere sulla terra un granello di sabbia. Gli uomini non ci fecero caso. Che cosa avrebbero significato cento, mille granelli di sabbia in un immenso giardino fiorito? Passarono gli anni e i peccati degli uomini aumentavano; torrenti di sabbia invasero il mondo. Nacquero così i deserti, che di giorno in giorno diventarono sempre più grandi. E Dio continua ancor oggi ad ammonire gli uomini dicendo loro: Non riducete il mio giardino fiorito in un immenso deserto!».

Questa amara parabola dipinge in modo illuminante la crisi del pianeta. Per fortuna, un rigurgito di pentimento e rimorso sta emergendo proprio sotto i termini di ecologia e sostenibilità, temi che sono stati finalmente messi in agenda non solo dalla Chiesa attraverso l'enciclica Caritas in veritate di Benedetto $\mathrm{XVI}^{25}$ e la Laudato si' di papa Francesco ${ }^{26}$, ma anche dagli Stati, dagli organismi internazionali e dalle stesse strutture economiche. A metà degli anni Settanta, attraverso un rapporto dell'allora Segretario Generale dell'ONU, Dag Hammarskjöld, si iniziò a sottoporre a critica il modello di sviluppo dominante. Fu, però, solo nel 1987 che la "Commissione mondiale

\footnotetext{
${ }^{25}$ Cf. BENEDETTO XVI. Lettera Enciclica Caritas in Veritate. Roma: Editrice Vaticana, 2009.

${ }^{26}$ Cf. FRANCESCO. Lettera Enciclica Laudato Si, sulla cura della casa comune. Roma: Editrice Vaticana, 2015.
} 
sull'ambiente e lo sviluppo" (nota come "Commissione Brundtland") definì in modo chiaro e ampio il concetto e il programma di sviluppo sostenibile, come «processo di cambiamento tale per cui lo sfruttamento delle risorse, la direzione degli investimenti, l'orientamento dello sviluppo tecnologico e i cambiamenti istituzionali siano coerenti coi bisogni futuri e non solo con gli attuali».

In quel testo si introducevano anche elementi etici e sociali per una «effettiva partecipazione dei cittadini nel processo decisionale e una maggior democrazia a livello di scelte internazionali», così che si potessero «soddisfare i bisogni fondamentali di tutti ed estendere a tutti la possibilità di attuare le proprie aspirazioni a una vita migliore». In questa linea, nel dicembre 2002, l'assemblea generale dell'ONU proclamò l'arco 2005-2014 come il "Decennio dell'educazione allo sviluppo sostenibile". Fondamentale - a livello socio-culturale generale - è far comprendere che la sostenibilità è uno dei diritti umani capitali. È noto, infatti, che ormai si è soliti elencare quattro "generazioni" di diritti.

I diritti di "prima generazione" sono quelli civili e politici (vita, dignità personale, libertà). Diritti di "seconda generazione" sono quelli economici, sociali, culturali descritti nella Dichiarazione universale dei diritti dell'uomo ${ }^{27}$ (1948), come salute, lavoro, istruzione e così via. Di "terza generazione" sono i diritti di solidarietà riguardanti soprattutto i soggetti più vulnerabili: pace, equilibrio ecologico, difesa ambientale e delle risorse nazionali, autodeterminazione dei popoli. Infine, alla "quarta generazione" appartengono i nuovi diritti relativi al campo delle manipolazioni genetiche, della bioetica e delle nuove tecnologie di comunicazione. È, dunque, importante inquadrare la sostenibilità nell'orizzonte più vasto della dignità umana, della morale sociale e degli stessi principi religiosi.

È ciò che è ribadito a più riprese nell'enciclica Laudato si' che per almeno una dozzina di volta usa la terminologia "sostenibile/sostenibilità". Papa Francesco lo fa partendo dalla Carta della Terra promulgata a L'Aja il 29 giugno 2000: «Come mai prima d'ora nella storia, il destino comune ci obbliga a cercare un nuovo inizio [...]. Possa la nostra epoca essere ricordata per il risveglio di una nuova riverenza per la vita, per la risolutezza nel raggiungere la sostenibilità, per l'accelerazione della lotta per la giustizia e la pace, e per

${ }^{27}$ Cf. Dichiarazione Universale dei Diritti Umani, Assemblea Generale delle Nazioni Unite, 10 dicembre 1948. 
la gioiosa celebrazione della vita ${ }^{28}$. Per questo «la sfida urgente di proteggere la nostra casa comune comprende la preoccupazione di unire tutta la famiglia umana nella ricerca di uno sviluppo sostenibile e integrale ${ }^{29}$.

Certo, il Papa è consapevole dei rischi che l'uso scontato e abitudinario del termine "sostenibilità", come abbiamo già sottolineato in apertura, e lo stesso «discorso della crescita sostenibile possa diventare spesso un diversivo e un mezzo di giustificazione che assorbe valori del discorso ecologista all'interno della logica della finanza e della tecnocrazia ${ }^{30}$. Per questo, è necessario sollecitare «una creatività capace di far fiorire nuovamente la nobiltà dell'essere umano, perché è più dignitoso usare l'intelligenza, con audacia e responsabilità, per trovare forme di sviluppo sostenibile ed equo, nel quadro di una concezione più ampia della qualità della vita ${ }^{31}$.

\subsection{Etica, teologia e sostenibilità}

Siamo partiti con la visione genesiaca della creazione; l'abbiamo riletta anche attraverso la concezione musulmana e abbiamo introdotto l'impegno che la Chiesa cattolica propone ai cristiani e anche a tutti gli uomini di buona volontà. Concludiamo questa considerazione essenziale sul nesso tra etica, teologia e sostenibilità, con una parabola moderna che è evocata dal filosofo Martin Heidegger in una delle sue opere più rilevanti, Essere e tempo ${ }^{32}$ (1927). Essa è la ricreazione libera di elementi mitici greci. Protagonista è una dea dal nome emblematico di "Cura", sinonimo del nostro vocabolo "Sostenibilità".

Attraversando un fiume, essa raccolse il fango della sponda e plasmò una figura umana. Giove le infuse lo spirito e la rese una creatura vivente. Cura e Giove si misero a litigare su chi avesse il diritto di imporre il nome e, quindi, il diritto di proprietà sulla persona umana. A questo punto reclamò il suo potere anche la dea Terra da cui quell'essere era stato tratto. I tre ricorsero a Saturno, il dio giudice che emise questa sentenza: «Tu, Giove, che hai dato lo spirito, al momento della morte riceverai lo spirito. Tu, Terra, che hai dato il corpo, riceverai il corpo. Ma finché la creatura umana vivrà, sarà sotto la tutela e la giurisdizione di Cura». Ecco perché la sostenibilità deve essere una

\footnotetext{
${ }^{28}$ FRANCESCO. Lettera Enciclica Laudato Sì, n. 207.

${ }^{29}$ FRANCESCO. Lettera Enciclica Laudato Si, n. 13.

${ }^{30}$ FRANCESCO. Lettera Enciclica Laudato Si, n. 194.

${ }^{31}$ FRANCESCO. Lettera Enciclica Laudato Si, n. 192.

${ }^{32}$ Cf. HEIDEGGER, M. Essere e tempo. Roma: Mondadori, 2011.
} 
sorta di grande protettrice che veglia sull'umanità, sulla sua storia e sulla sua evoluzione.

\section{Riferimenti bibliografici}

ADORNO, T. W. Minima Moralia. Meditazioni della vita offesa. Torino: Einaudi, 2005.

BAILONE, G. Plotino. Pistoia: Editrice Petite Plaisance, 2010.

BARZAGHI, G. (Curatore). Tommaso d'Aquino (san), Somma Teologica. Edizione Integrale: Opera completa in 4 volumni. Bologna: Edizione Studio Domenicano, 2014.

BENEDETTO XVI. Lettera Enciclica Caritas in Veritate. Roma: Editrice Vaticana, 2009.

BIEIRWALTES, W. Agostino e il Neoplatonismo Cristiano. Milano: Vita e Pensiero, 1991.

BUBER, M. Il problema dell'uomo. Genova: Marietti, 2013.

CAMPANINI, G. (Curatore). Emmanuel Mounier, Il Personalismo. Roma: AVE, 2004.

CAMPI, R. (Curatore). René Descartes, Discorso del Metodo. Milano: Rizzolli, 2010.

CENTI, T. S. (Curatore). Tommaso d'Aquino (san), Somma contro i gentili. 3 volumi. Bologna: Edizione Studio Domenicano, 2001.

Dichiarazione Universale dei Diritti Umani. Assemblea Generale delle Nazioni Unite, 10 dicembre 1948.

FIORENTINO, F. (Curatore). Tommaso d'Aquino, Sulla Verità. Milano: Bompiani, 2005.

FOUCAULT, M. Il coraggio della verità. Roma: Feltrinelli, 2011.

FRANCESCO. Lettera Enciclica Laudato Sì, sulla cura della casa comune. Roma: Editrice Vaticana, 2015.

GENTILE, G. (Traduttore). Immanuel Kant, Critica della ragion pratica. Testo originale a fronte. Bari: Laterza, 2005.

GHIA, F. Max Weber, Il Politeismo dei valori. Brescia: Morcelliana, 2010. 
HARDING, S. Science and social inequality, Feminism and Postcolonial Issues. Chiacago: University Ilinois Press, 2006.

HEIDEGGER, M. Essere e tempo. Roma: Mondadori, 2011.

La Bibblia di Gerusalemme. Bologna: Dehoniane, 2014.

MATI, S. (Curatore). Platone, Fedro. Roma: Feltrinelli, 2008.

MAZZARELLI, C. (Curatore). Aristotele, Etica Nicomachea. Milano: Bompiani, 2000.

MOSCATO, A. (Curatore). Emmanoel Levinás, L'umanesimo dell 'altro uomo. Genova: Il Nuovo Melangolo, 1998.

MUSIL, R. L'uomo senza qualità. Torino: Einaudi, 2005.

REALE, G. (Curatore). Aristotele, Metafisica. Milano: Bompiani, 2000.

REALE, G. (Curatore). Platone, Apologia di Socrate. Roma: Feltrinelli, 2000.

REALE, G. (Curatore). Platone, Opere Complete, Tutti gli scritti. Milano: Bompiani, 2000.

RICOEUR, P. La persona. Brescia: Morcelliana, 1997.

RICOEUR, P. Sé come un altro. Milano: Jaca Book, 2011.

RIST, J. M. Agostino, Il Battesimo del Pensiero Antico. Milano: Vita e Pensiero, 1997.

SANTI, R. (Curatore). Tommas Hobbes, Leviatano. Milano: Bompiani, 2001. WITTGENSTEIN, L. Tractatus logico-philosophicus. Roma: Carocci, 2012.

\section{Cardinal Gianfranco Ravasi}

Teologo, Biblista ed Ebraista, italiano, Arcivescovo Cardinale, dal 2007 è presidente del Pontificio Consiglio della Cultura, della Pontificia Comissione di Archeologia Sacra e del Consiglio di Coordinamento fra Accademie Pontificie Itália

Recebido em: 07/06/16

Aprovado em: 08/07/16 